\title{
EFECTO DE LA COMPOSICIÓN QUÍMICA DEL BAÑO EN LA MICROESTRUCTURA Y RESISTENCIA A LA CORROSIÓN DE LOS RECUBRIMIENTOS DE ZINC POR INMERSIÓN EN CALIENTE: UNA REVISIÓN \\ Effect of Chemical Bath Composition on Microstructure AND CORRosion Resistance of Zinc Coatings by Hot Dip: A REVIEW
}

\author{
Jeanette Hernández ${ }^{1, *}$, Maribel Suárez ${ }^{2}$
}

\section{Resumen}

Los recubrimientos metálicos son métodos ampliamente utilizados para la protección contra la corrosión de aleaciones metálicas, siendo el proceso de cincado por inmersión en caliente uno de los que presenta mayor evolución a nivel industrial. El objetivo de este trabajo es realizar una revisión bibliográfica sobre la influencia de la adición de elementos aleantes en el baño, en la microestructura y en el comportamiento a la corrosión de recubrimientos de zinc obtenidos por la técnica de inmersión en caliente. Se estableció que la composición química de los baños galvanizados influye en las características microestructurales de los recubrimientos y en su comportamiento a la corrosión. La mejora de la resistencia a la corrosión de los recubrimientos de zinc se produce por la adición a los baños de elementos generalmente más activos que el zinc, tales como el magnesio o el aluminio que permiten la formación de capas pasivas que retardan el proceso corrosivo.

Palabras clave: zinc, corrosión, microestructura, recubrimiento, galvanizado, inmersión en caliente.

\section{Abstract}

Metal coatings are widely used methods for corrosion protection of metal alloys, the hot dip galvanizing process being one of the most industrially evolved. The objective of this work is to carry out a literature review, on the influence of the addition of alloying elements in the bathroom, on the microstructure and on the corrosion behavior of zinc coatings obtained by hot dipping technique. It was determined that the chemical composition of the galvanized baths influences the microstructural characteristics of the coatings and their corrosion behavior. The improvement of the corrosion resistance of zinc coatings is produced by the addition to the baths of elements generally more active than zinc, such as magnesium or aluminum that allow the formation of passive layers that retard the corrosive process.

Keywords: Zinc, Corrosion, Microstructure, Coating, Galvanized, hot dip.

\footnotetext{
$\overline{1, *}$ Departamento de Ingeniería Metalúrgica, Universidad Nacional Experimental Politécnica «Antonio José de Sucre»

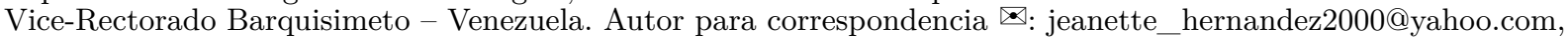
jphernandez@unexpo.edu.ve (D) http://orcid.org/0000-0002-8359-1058

${ }^{2}$ Escuela de Ingeniería Metalurgia y Ciencia de los Materiales, Facultad de Ingeniería, Universidad Central de Venezuela, Caracas - Venezuela. (D) http://orcid.org/0000-0003-0449-0417
}

Recibido: 21-09-2019, aprobado tras revisión: 08-11-2019

Forma sugerida de citación: Hernández, J. y Suárez, M. (2020). «Efecto de la composición química del baño en la microestructura y resistencia a la corrosión de los recubrimientos de zinc por inmersión en caliente: Una revisión». InGEnIUS. N. ${ }^{\circ} 23$, (enero-junio). pp. 40-52. DOI: https://doi.org/10.17163/ings.n23.2020.04. 


\section{Introducción}

Los procesos para obtener recubrimientos mediante tecnologías de inmersión en caliente y en particular los de zinc puro, se vienen usando como protección contra la corrosión ante diferentes ambientes, especialmente urbanos, en los que su comportamiento resulta muy satisfactorio en términos generales. Sin embargo, en ambientes marinos, en los que la humedad y la concentración de cloruro de sodio suelen ser elevados, la resistencia a la corrosión de los aceros recubiertos con zinc puro puede disminuir sensiblemente [1].

Algunas propuestas de solución a esta limitación incluyen la generación de recubrimientos base zinc con la adición de algunos elementos aleantes, siendo los más estudiados el aluminio, el magnesio, el silicio, el cobre, el estaño, el níquel y otros elementos en menor proporción $[1,2]$.

Desde un punto de vista tecnológico, los parámetros operacionales para efectuar el proceso de recubrimiento de zinc sobre el acero por la técnica de inmersión en caliente se han mantenido prácticamente invariables con el tiempo, pero las nuevas aplicaciones en la industria automotriz y en la construcción han originado que, en la actualidad, exista una cantidad considerable de trabajos de investigación acerca de los aspectos del proceso y de nuevos tipos de recubrimientos de zinc $[3,4]$, lo cual ha aumentado los estudios de la influencia de agregar elementos aleantes al baño de zinc, la microestructura y las propiedades de los recubrimientos obtenidos $[3,4]$.

En vista de lo anterior, este trabajo tiene como objetivo realizar una revisión bibliográfica, sobre la influencia de la adición de elementos aleantes en el baño, en la microestructura y en el comportamiento a la corrosión de recubrimientos de zinc obtenidos por la técnica de inmersión en caliente.

\section{Desarrollo de recubrimientos de zinc sobre acero}

La Asociación Americana de Galvanizadores [5] indica que la historia registrada de la galvanización se remonta al año 1742 cuando el químico francés llamado Melouin, en una presentación ante la Academia Francesa Royal, describe un método de recubrimiento de hierro por inmersión en zinc líquido.

En el año 1836, Sorel, otro químico francés, obtuvo una patente para fabricar un recubrimiento de zinc sobre hierro, después de efectuar una limpieza con $9 \%$ de ácido sulfúrico y usando un fundente con cloruro de amonio.

Para el año 1850, la industria británica de galvanizado utilizaba 10000 toneladas de zinc al año para la protección del acero y en 1870, se instaló la primera planta de galvanizado en los Estados Unidos.
Los recubrimientos obtenidos por la técnica de galvanizado, son ampliamente utilizados en diversas industrias. Las empresas de servicios públicos, procesos químicos, pulpa y papel, industria automotriz y transporte, históricamente han hecho un uso extensivo de la galvanización para el control de la corrosión, y siguen haciéndolo hoy en día. Durante más de 150 años, la galvanización ha tenido una probada historia de éxito comercial como un método de protección a la corrosión en una gran variedad de aplicaciones en todo el mundo. Las investigaciones en los últimos años, indican que los estudios se han centrado en la influencia de la composición química del baño en la microestructura y las propiedades de los recubrimientos galvanizados. Los cambios en la composición química de los baños de zinc buscan, sin duda alguna una mejora significativa en las propiedades del recubrimiento galvanizado, bien sea, en la resistencia a la corrosión, en los procesos de soldabilidad, y/o en las propiedades mecánicas del mismo.

En la Figura 1, se presenta un recubrimiento típico de acero galvanizado, donde se puede observar tres capas bien definidas, las cuales se forman durante el proceso de galvanizado, cada capa corresponde a la formación de productos intermetálicos y de acuerdo con su composición se conocen como Gamma, Delta y Zeta y la capa superior de zinc puro Eta. Durante el proceso de galvanización, las capas se desarrollan naturalmente durante una reacción metalúrgica entre el hierro del acero y el zinc del baño [5].

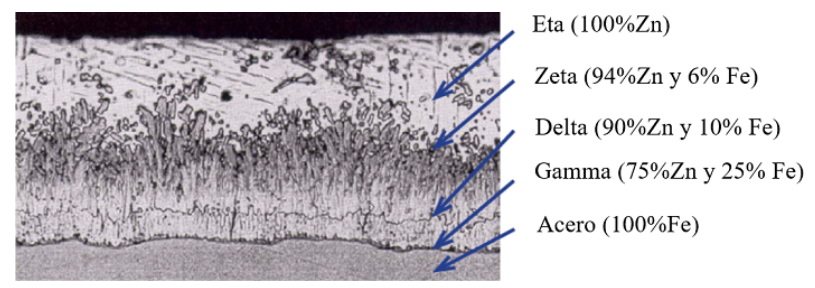

Figura 1. Microestructura de un recubrimiento típico de $\mathrm{Zn}$ mostrando las capas formadas con su respectiva composición química. [5]

Las investigaciones se han orientado a estudiar la metalurgia del acero galvanizado desde un punto de vista científico para desarrollar relaciones a las aplicaciones prácticas. Estos estudios involucran la evaluación sistemática del proceso de inmersión en caliente, junto con los diagramas de equilibrio o de fases Fe-Zn y la cinética de formación de las capas del recubrimiento de Zn [3].

Cabe destacar, que el aluminio es uno de los elementos más utilizados en el baño de zinc. El desarrollo comercial de estos recubrimientos, tales como el Galvalume ${ }^{\circledR}(\mathrm{Zn}-55 \mathrm{Al})$ en 1970 y del Galfan ${ }^{\circledR}$ (Zn-5Al) durante 1980, demostraron con éxito que la incorporación del aluminio mejora significativamente la resistencia a la corrosión del recubrimiento base zinc. 
Desde entonces se han efectuado numerosas investigaciones acerca de los recubrimientos $\mathrm{Zn}-\mathrm{Al}$ aplicados por inmersión en caliente sobre el acero.

El Galvalume ${ }^{\circledR}$, es un recubrimiento de aproximadamente $55 \%$ de aluminio que contiene aproximadamente $1,5 \%$ de silicio, el cual es añadido con el propósito de prevenir una reacción exotérmica en la intercara recubrimiento / sustrato de acero. Durante el proceso se generan en la intercara, compuestos intermetálicos Fe-Zn-Al. La superficie del Galvalume ${ }^{\circledR}$ contiene características de lentejuelas, que consisten en dendritas de aluminio con distancia del brazo dendrítico claramente medibles.

El Galfan ${ }^{\circledR}$ es un recubrimiento de zinc con $5 \%$ de aluminio, que está cerca del punto eutéctico, tienen una excelente conformabilidad y protección a la corrosión. Dos composiciones se han reportado sobre la base de adiciones a la composición eutéctica: pequeñas adiciones que contiene lantano y cerio hasta alrededor del 0,5\% y adiciones de 0,5\% de magnesio. Estas adiciones, se hacen para mejorar la humectabilidad o mojabilidad del baño fundido sin reducir la resistencia a la corrosión del recubrimiento. La microestructura del Galfan ${ }^{\circledR}$ se caracteriza por una estructura bifásica, la fase proeutectoide eta $(\eta)$ rica en zinc rodeada por una fase de tipo eutéctica formada por beta $(\beta)$ y eta $(\eta)$. Sin embargo, la microestructura puede variar dependiendo de velocidad de enfriamiento [3].

En la Figura 2 se observa este último efecto, donde Rico y Hernández [6] estudiaron la influencia de la velocidad de enfriamiento en la morfología y distribución de las fases de los recubrimientos Zn-7Al sobre acero. La microestructura del recubrimiento enfriado en agua muestra la mezcla eutéctica segregada en la intercara recubrimiento/acero, y los enfriados dentro del horno, presentan un microconstituyente monotectoide de morfología dendrítica.

Según el ASM Handbook: Corrosion [7] hay buena evidencia de que pequeñas cantidades de elementos de aleación pueden afectar la resistencia a la corrosión de los recubrimientos galvanizados. Los mecanismos para estos efectos son complejos y no se entienden completamente, pero la literatura sugiere que los elementos de aleación se segregan a los límites de grano, donde pueden acelerar o retardar el ataque intergranular. Considerando, la aleación $\mathrm{Zn} / 0,1-0,2 \mathrm{Al}$, típicamente utilizada para galvanizado en caliente, se encontró que el agregado en el baño de magnesio y cobre reduce el mecanismo de corrosión intergranular, mientras que la presencia de bismuto, cadmio, plomo y estaño aumenta el ataque localizado. También hay algunas evidencias de que la adición de níquel al baño reduce la reacción de evolución de hidrógeno, y aumenta la resistencia a la corrosión en ambientes que contengan cloruros $[8,9]$.

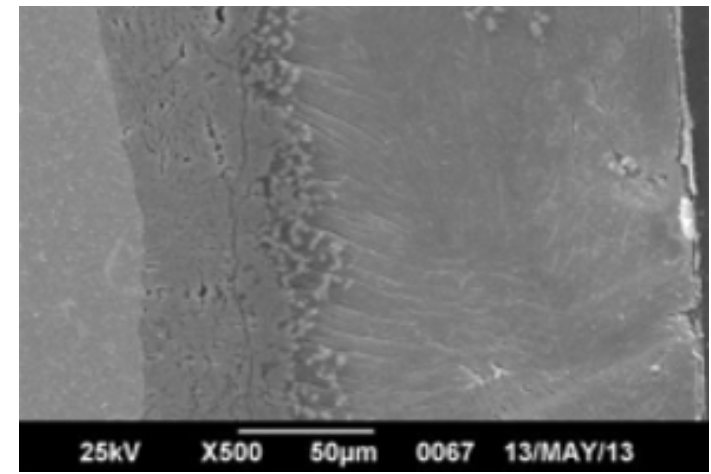

(a)

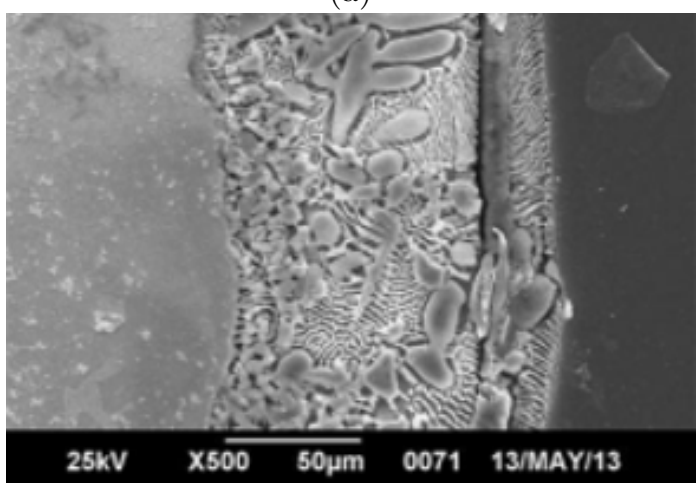

(b)

Figura 2. Micrografías por microscopía electrónica de barrido (MEB) de recubrimientos $\mathrm{Zn}-7 \mathrm{Al}$, con diferentes condiciones de enfriamiento. a) agua. b) horno [6].

Recientemente, los estudios sobre los recubrimientos de Zn-Al-Mg han llamado la atención. La razón de su estudio sistemático masivo es su rendimiento en medios o ambientes salinos donde se han obtenido mejores comportamientos a la corrosión del orden de 10 a 20 veces respecto al recubrimiento de $\mathrm{Zn}$ y de 2 a 5 respecto a los de $\mathrm{Zn}$-Al. También se han encontrado otras propiedades importantes tal como la capacidad de autocuración, por lo que el área de la incisión puede volver a cerrarse y protegerse, resistencia al rayado y al desgaste, densidad del recubrimiento más ligero, entre otras ventajas [10].

Compañías japonesas, europeas y sudafricanas ya han desarrollado recubrimientos $\mathrm{Zn}-\mathrm{Al}-\mathrm{Mg}$, tal como la Nisshin Steel Co, que ha patentado la marca ZAM®. Sin embargo, aún falta información detallada sobre el mecanismo anticorrosivo del recubrimiento de $\mathrm{Zn}-\mathrm{Mg}-\mathrm{Al}$ en la literatura abierta, y se sabe muy poco específicamente sobre el rendimiento electroquímico del recubrimiento de $\mathrm{Zn}-\mathrm{Al}-\mathrm{Mg}$. Por otra parte, no se han optimizado las condiciones de operación del proceso de inmersión en caliente de tal forma de obtener una superficie lisa del recubrimiento $\mathrm{Zn}-\mathrm{Al}-\mathrm{Mg}$, siendo un desafío en este campo [10].

Li et al. [2] evaluaron un recubrimiento sobre acero de aleación Zn-5Al-0,5Mg-0,08Si y fue comparado con el recubrimiento Zn-5Al. Encontraron que la resisten- 
cia a la corrosión del recubrimiento Zn-5Al no es muy alta en comparación con el que contiene magnesio y silicio.

En la Figura 3 se muestra la morfología de la superficie del recubrimiento, de $\mathrm{Zn}-5 \mathrm{Al}$ y los resultados correspondientes a la composición química en los puntos A y B. El recubrimiento consiste en $\beta$ (Zn) grueso y fases eutécticas binarias de $\mathrm{Zn}-\mathrm{Al}$.

Debido a la diferencia de potencial entre las dos fases (Figura 3), cuando se coloca el recubrimiento en el ambiente corrosivo, la fase de potencial más electronegativo acelerará el proceso de corrosión, mientras que la fase de potencial menos electronegativo estará protegida de corroerse.

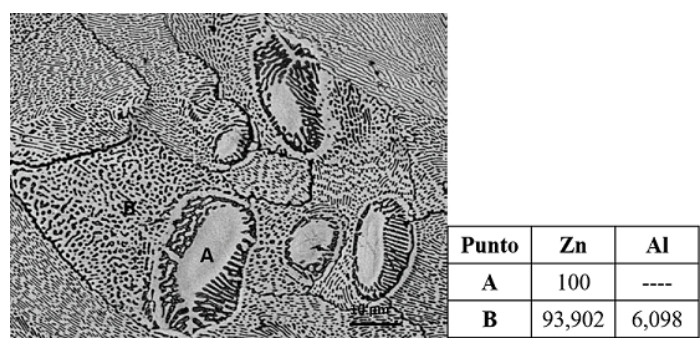

Figura 3. Micrografía por microscopía electrónica de barrido (MEB) de un recubrimiento de $\mathrm{Zn}-5 \mathrm{Al}$ y la composición química en porcentaje en peso por espectroscopía de energía dispersa de rayos X (EDS) en los puntos A y B. [2]

La Figura 4 indica la imagen por microscopía electrónica de barrido (MEB) de un recubrimiento de Zn-5Al-0,5Mg-0,08Si. Se puede ver que la separación del brazo dendrítico de $\mathrm{Zn}-5 \mathrm{Al}$ del recubrimiento se reduce significativamente de $\sim 10 \mu \mathrm{m}$ a $2-5 \mu \mathrm{m}$, después de agregar elementos de aleación $\mathrm{Mg}$ y Si. Se forman las fases $\beta(\mathrm{Zn})(\mathrm{A})$, otra rica en $\mathrm{Al}(\mathrm{B})$ y un eutéctico terciario $\mathrm{MgZn}_{2}-\mathrm{Zn}-\mathrm{Al}$ (C) [2]. El magnesio es solo distribuido en los límites de granos, y esto puede hacer que los granos sean más finos e impedir la corrosión intergranular.

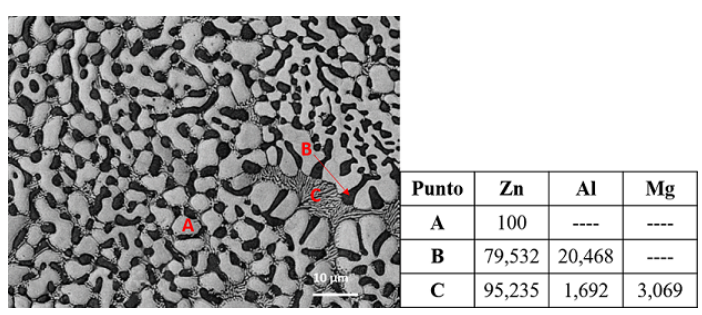

Figura 4. Micrografía por microscopía electrónica de barrido (MEB) de un recubrimiento de $\mathrm{Zn}-5 \mathrm{Al}-0,5 \mathrm{Mg}-0,08 \mathrm{Si}$ y la composición química en porcentaje en peso por espectroscopía de energía dispersa de rayos X (EDS) en los puntos A y B. [2]

En este mismo sentido, Schuerz et al. [11], efectuaron un estudio sobre chapas de acero recubiertas convencionalmente por inmersión en caliente de Zn (Z) y de aleación Zn-Al-Mg (ZM), con una composición química que se indica en la Tabla 1.

Tabla 1. Composición química de los recubrimientos evaluados en porcentaje en peso [11]

\begin{tabular}{cccc}
\hline Recubrimiento & Zn & Al & Mg \\
\hline $\mathrm{Z}$ & 99,8 & 0,2 & \\
$\mathrm{ZM}$ & 96 & 2 & 2 \\
\hline
\end{tabular}

La microestructura de los recubrimientos convencionales de zinc $(\mathrm{Z})$ y cinc-aluminio-magnesio (ZM) difieren considerablemente como se ilustra en la Figura 5 .

El recubrimiento $\mathrm{Z}$ consiste en una capa de material homogéneo de zinc puro. El aluminio está enriquecido en la intercara acero/recubrimiento en forma de una capa muy delgada de un compuesto intermetálico $\mathrm{Al}-\mathrm{Fe}$, lo que asegura una correcta adherencia del recubrimiento sobre la superficie de acero. Por otro lado, el recubrimiento ZM consta de diferentes fases: una fase de zinc, que también incluye pequeñas cantidades de aluminio, una fase de aluminio y la fase de $\mathrm{MgZn}_{2}$. Este último y el zinc también se presentan en forma de eutéctico binario (marcado con un rectángulo), así como un eutéctico terciario de zinc, aluminio y la fase $\mathrm{MgZn}_{2}$ (marcado con un marco óvalo).

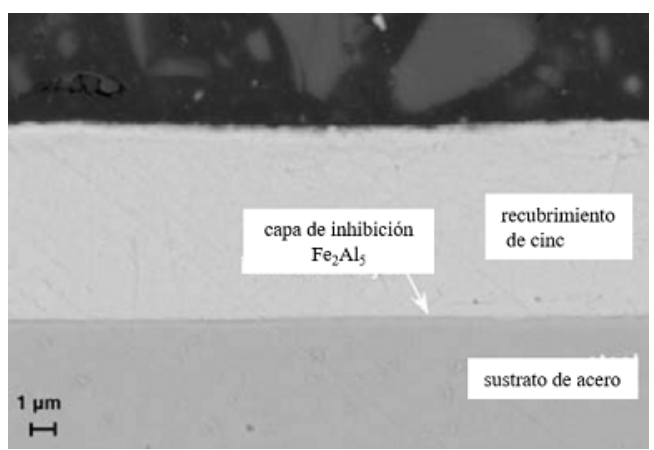

(a)

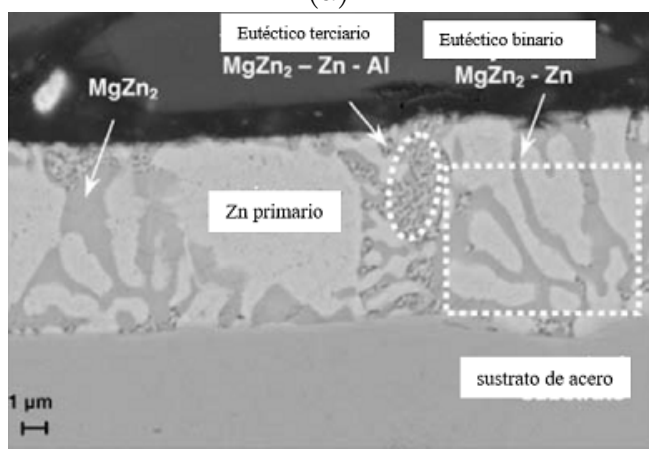

(b)

Figura 5. Micrografía por microscopía electrónica de barrido (MEB) de un recubrimiento de $\mathrm{Zn}$ convencional $\mathrm{Z}$ (a) y de un recubrimiento de aleación Zn-Al-Mg ZM (b). [11] 
El recubrimiento metálico ZM se convierte en un material estable, adherente y una capa de óxido rica en aluminio que protege el sustrato de acero en ambientes corrosivos. Esta capa es la razón principal de la resistencia a la corrosión mejorada del recubrimiento $\mathrm{ZM}$ en un entorno que contiene cloruro de sodio.

Otros desarrollos que han surgido como innovación de los procesos de cincado por inmersión caliente son los procesos de doble inmersión, los cuales se plantearon basándose en los problemas operacionales que presentan durante la inmersión en caliente los recubrimientos $\mathrm{Zn}-\mathrm{Al}$, ya que no tienen buena compatibilidad con los sistemas típicos de flux y el utilizar otros no lo hace práctico en la industria de la galvanización. Además de que la cantidad de aluminio que entra en la capa del recubrimiento representa un problema adicional en la composición del baño, ya que se reduce considerablemente la concentración de aluminio, lo que genera un continuo monitoreo [4].

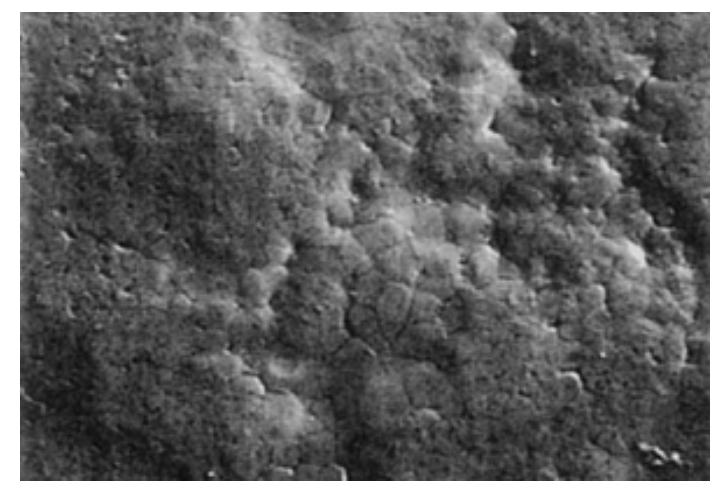

(a)

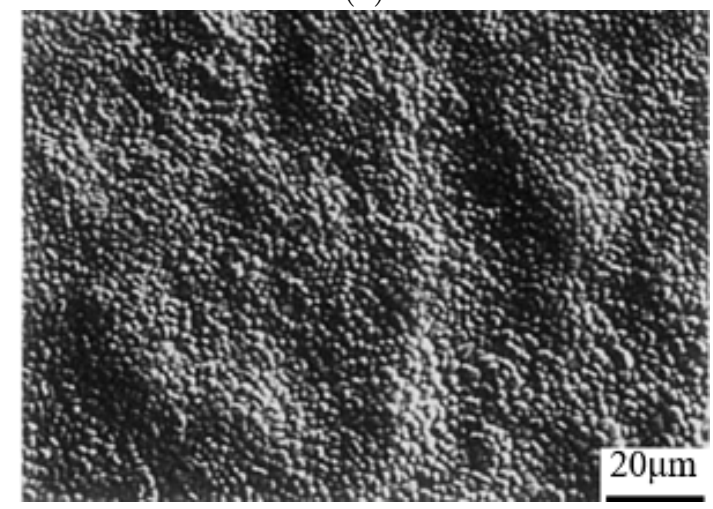

(b)

Figura 6. Morfología superficial por MEB de los recubrimientos formados sobre aceros de bajo carbono por inmersión en caliente en dos etapas Z6A (a) y Z6AMS (b). [12]

En este sentido, Tanaka et al. [12] evaluaron recubrimientos sobre acero con bajo contenido de carbono por inmersión en caliente en dos etapas (doble inmersión), principalmente en un baño de Zn y luego en un baño de Zn-6Al (Z6A) con o sin adición de 0,5 \% en peso de Mg y $0,1 \%$ en peso de Si (Z6AMS). En la Figura 6 se muestra la morfología de la superficie de los recubrimientos formados por la inmersión en caliente de dos etapas con baños secundarios de Z6A (a) y Z6AMS (b). La superficie del revestimiento formado en el Z6AMS mostró granos muy finos y pocos defectos en comparación con el Z6A.

Las capas de recubrimiento consistían en una estructura dúplex; una capa externa y una capa interna de aleación (Figura 7). Identificaron que la capa de aleación interna estaba compuesta principalmente de una fase intermetálica de $\mathrm{Fe}_{4} \mathrm{Al}_{13}-\mathrm{Zn}$. Observaron que los cristales de esta fase son distribuidos aleatoriamente en el recubrimiento Z6A, mientras que el recubrimiento Z6AMS tiene una estructura columnar con crecimiento perpendicular al sustrato de acero.

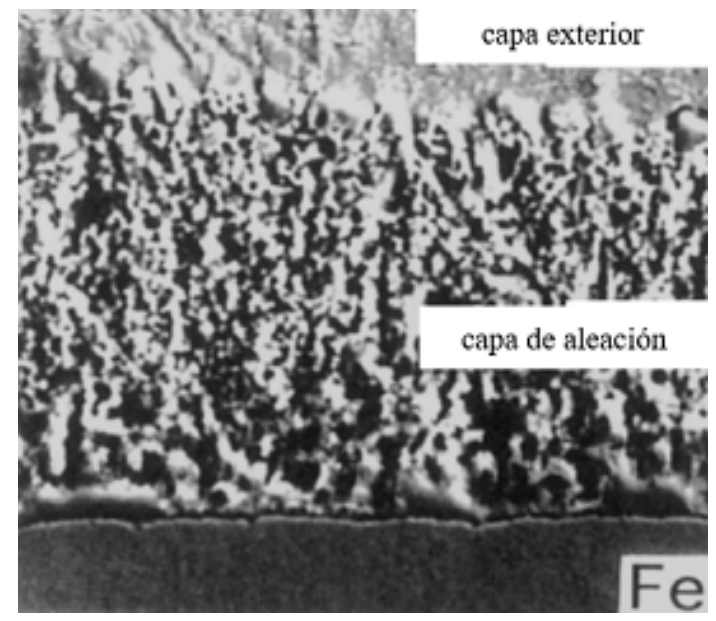

(a)

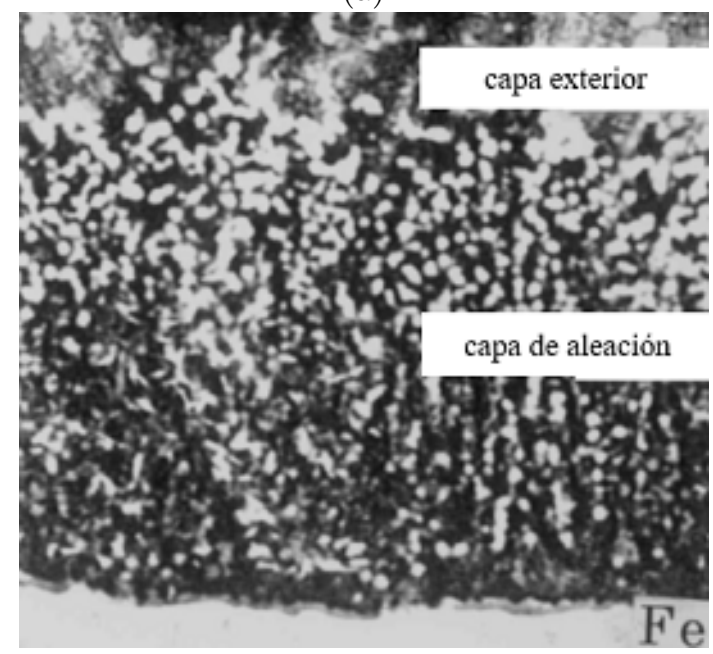

(b)

Figura 7. Microestructuras de las secciones transversales de los recubrimientos Z6A (a) y Z6AMS (b). [12]

Las Figuras 8 (a) y (b) muestran la capa externa para los recubrimientos Z6A y Z6AMS, respectivamente. El área oscura es principalmente una fase $\alpha$ - $\mathrm{Al}$ y el área clara es la estructura de un eutéctico de Zn (Al) y $\alpha$-Al. Se encontró que el $\alpha$-Al en el recubri- 
miento Z6A en la Figura 8 (a) era mucho más grueso que el del recubrimiento Z6AMS en la Figura 8 (b). El recubrimiento Z6AMS se corroyó lentamente y de forma homogénea, mientras que el recubrimiento de Z6A se degrada localmente debido a una corrosión preferencial a lo largo de defectos. El recubrimiento Z6AMS presenta Mg y Si en la capa externa y $\mathrm{Si}$ en la capa de aleación interna, lo cual lo que hace más anticorrosivo.

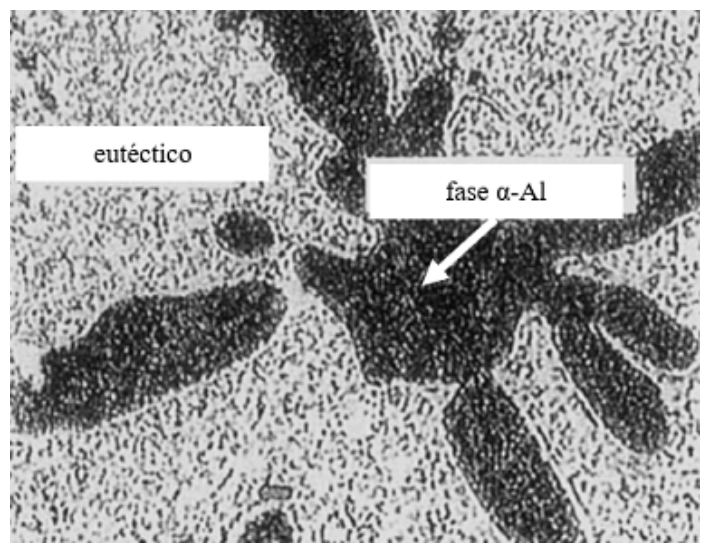

(a)

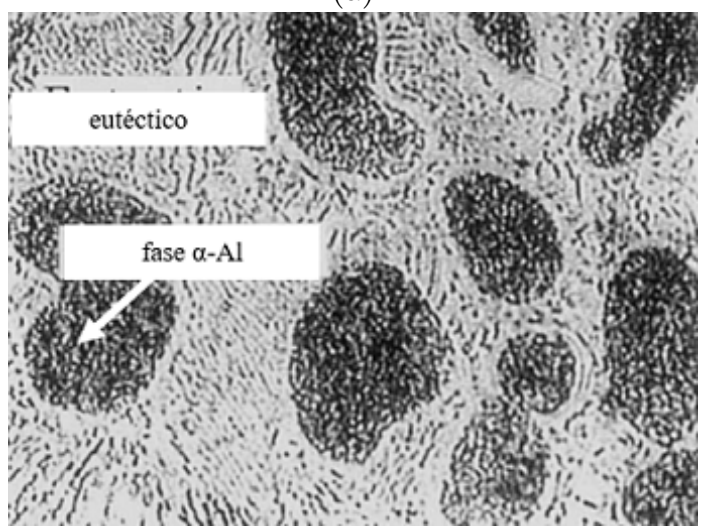

(b)

Figura 8. Microestructuras de la capa externa de los recubrimientos Z6A (a) y Z6AMS (b). [12]

En los procesos de doble inmersión también se destaca el estudio efectuado por Tachibana y colaboradores [13], donde se evaluó un nuevo recubrimiento por inmersión en caliente de $\mathrm{Zn}_{7} \mathrm{Al}$ sobre un acero estructural el cual fue aplicado mediante una doble capa $\mathrm{Zn}-\mathrm{Zn}_{7} \mathrm{Al}$, para evitar la corrosión severa en las zonas costeras. El recubrimiento doble fue significativamente eficaz en la prevención de la corrosión, particularmente en la orilla del mar. Se estimó que la vida del recubrimiento $\mathrm{Zn}-\mathrm{Zn}_{7} \mathrm{Al}$ sobre el acero fue cuatro veces la del acero recubierto con Zn en la orilla del mar. Se reveló que la excelente adhesividad del doble revestimiento $\mathrm{Zn}-\mathrm{Zn}_{7} \mathrm{Al}$ sobre el acero fue debido a la formación de la región de intercara que consta de una fase heterogénea fina de una mezcla de cinc, aluminio y hierro.
También Rico y Carrasquero [8] realizaron una caracterización microestructural de recubrimientos de zinc por el método de doble inmersión sobre acero. La temperatura del baño de inmersión fue de $550{ }^{\circ} \mathrm{C}$ y los recubrimientos se realizaron usando diferentes tiempos de inmersión. Todos los recubrimientos mostraron tres zonas definidas. La primera zona, que corresponde a la interfaz acero/revestimiento, presenta propiedades típicas de un proceso de galvanización tradicional a altas temperaturas. En la zona II, se observó una microestructura muy heterogénea; la zona III mostró una matriz de $\mathrm{Zn}-\mathrm{Fe}$ con partículas de compuestos de $\mathrm{Fe}_{2} \mathrm{Al}_{5} \mathrm{Zn}_{0,4}$, tal como se observa en la Figura 9. Las propiedades microestructurales de los recubrimientos fueron similares durante los tres tiempos de inmersión. Determinaron la presencia de $\mathrm{Fe}$ y $\mathrm{Al}$ en todo el recubrimiento, debido a la difusión del hierro del metal base y el aluminio que presenta el segundo baño de inmersión.

Así se tiene, que el desarrollo de los recubrimientos de zinc por inmersión en caliente se ha basado en modificar la composición química del baño, lo cual implica cambios microestructurales que mejoran sus propiedades para una aplicación específica. Los elementos de aleación que más se han estudiado con el fin de establecer su influencia en las propiedades generales de estos recubrimientos son el aluminio [3,6,13-17], el magnesio [2,10-12,16-19] y el silicio [2]. Otros elementos que en menor proporción se han estudiado son el cobre [1,20], el titanio, el níquel [21,22], etc.

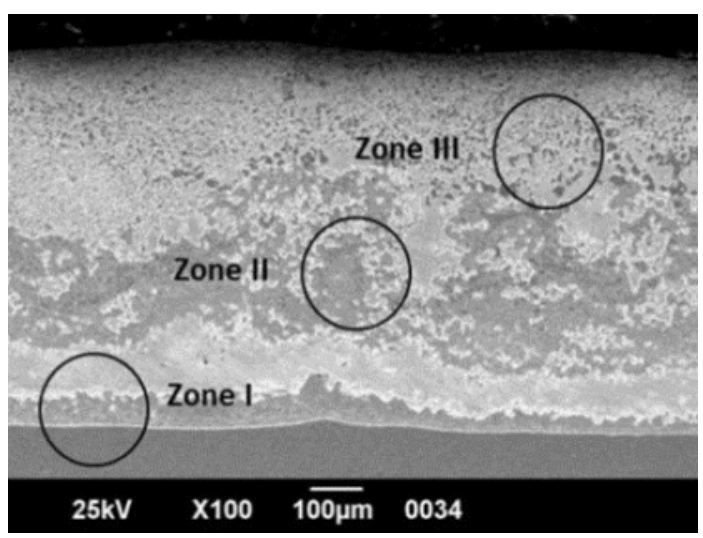

Figura 9. Micrografía MEB del recubrimiento de Zn/Zn$5 \mathrm{Al}$, con un tiempo de inmersión total de $120 \mathrm{~s}$, donde se muestran las tres zonas del recubrimiento. [8]

\section{Comportamiento a la corrosión de los recubrimientos de zinc}

Los recubrimientos de zinc añaden resistencia a la corrosión al acero de varias maneras; una como una capa barrera, ya que el recubrimiento continuo separa el acero del ambiente corrosivo; otra por protección 
galvánica, ya que el zinc actúa como un ánodo de sacrificio que protege el acero en áreas adyacentes a los huecos, rasguños y bordes del recubrimiento. El comportamiento del zinc se puede ver en una serie galvánica donde el zinc es menos noble que el acero en la mayoría de los medios a temperatura ambiente [3].

Una capa superficial porosa de óxido de zinc se forma en la superficie por un mecanismo de disolución/reprecipitación, lo que conduce a vías de corrosión preferenciales en el área de alta porosidad o discontinuidades, lo que explica la tasa de corrosión lineal. Además, después de la disolución del zinc metálico, el hidróxido de zinc puede precipitar a las áreas catódicas del acero expuesto, formando una capa barrera secundaria. Por lo que el recubrimiento de zinc se corroerá a una velocidad más lenta que el sustrato de acero, aunque la tasa de corrosión del zinc variará dependiendo del ambiente de exposición [3].

En el caso de recubrimientos $\mathrm{Zn}-\mathrm{Al}$ como el Galfan ${ }^{\circledR}$, la corrosión en ambientes no marinos ocurre en dos etapas: protección temporal por formación de capa pasiva de óxido de aluminio y la acción galvánica del zinc donde se forma sulfato de zinc sobre la capa de óxido. La cinética es controlada por la segunda etapa por la necesidad de difundir a través de la capa de óxido, lo cual explica la disminución de la velocidad de corrosión del Galfan ${ }^{\circledR}$ respecto a los recubrimientos de zinc convencionales. También se ha demostrado que la fase rica en zinc ( $\eta$-eta) se corroe preferencialmente respecto al proeutéctico y eutéctico. Las adiciones de $\mathrm{Mg}$ (hasta $0,8 \%$ en peso) aumentan la cantidad de proeutéctico de fase $\mathrm{Al}$, lo cual mejora la resistencia a la corrosión. El Galfan ${ }^{\circledR}$ se corroe de forma parabólica mientras los recubrimientos convencionales se corroen en forma lineal [3].

La corrosión atmosférica del recubrimiento Galvalume® se lleva a cabo en las regiones interdendríticas ricas en zinc, lo que permite una protección galvánica. Luego los productos de corrosión quedan atrapados en esos espacios y actúan como una barrera a la corrosión. El proceso corrosivo es del tipo parabólico [3].

En vista de la importancia de evaluar sistemáticamente el comportamiento a la corrosión de estos recubrimientos en diferentes ambientes, múltiples investigadores se han abocado a realizar ensayos de corrosión acelerados, los tradicionales de exposición a condiciones atmosféricas, por inmersión y en cámara de niebla salina para conocer la velocidad de corrosión, así como las técnicas electroquímicas que permiten conocer mayor información sobre el mecanismo y la cinética de la corrosión. Estos estudios normalmente se efectúan de manera comparativa para los diferentes recubrimientos, de forma de establecer analogías y diferencias que permitan a la industria decidir sobre la aplicabilidad de cada uno de los recubrimientos evaluados.
En la Tabla 2 se observa un resumen de las técnicas utilizadas y los ambientes en los cuales generalmente son evaluados los diferentes recubrimientos base zinc.

Por otra parte, el estudio del comportamiento a la corrosión de los recubrimientos de zinc depende de muchos factores, pero la orientación más común de las investigaciones es relacionar los cambios microestructurales de los recubrimientos con su resistencia a la corrosión, por lo cual el añadir elementos aleantes a los baños de zinc tradicionales permiten obtener esos cambios. También en estas investigaciones se torna importante establecer los mecanismos que rigen los procesos corrosivos, basados en técnicas que permiten la caracterización de los productos de corrosión, para relacionarlas con las especies presentes en el ambiente y los elementos que conforma el material $[9,15,18,19,23,24]$.

En este sentido, Hosking et al. [25] investigaron acerca del comportamiento a la corrosión de los recubrimientos $\mathrm{Zn}-\mathrm{Mg}$ en comparación con recubrimientos convencionales de cinc. Para ello siguieron un programa experimental donde efectuaron ensayos de corrosión en una solución acidificada de $\mathrm{NaCl}$, caracterizaron los productos de corrosión y establecieron una propuesta del mecanismo del proceso corrosivo. Los resultados revelaron un aumento de tres veces la resistencia a la corrosión de los recubrimientos $\mathrm{Zn}-\mathrm{Mg}$ respecto a los convencionales de zinc; así como que la causa de esta mejora es la precipitación de simonkolleita $\left(\mathrm{Zn}_{5} \mathrm{Cl}_{2}(\mathrm{OH})_{8} \cdot \mathrm{H}_{2} \mathrm{O}\right)$ insoluble posiblemente modificada con magnesio absorbido, hidróxido de magnesio $\left(\mathrm{Mg}(\mathrm{OH})_{2}\right)$ y una especie de hidroxicarbonato, sobre la superficie corrosiva, retardando así las reacciones de corrosión.

Asimismo, Yao et al. [10] evaluaron el comportamiento a la corrosión comparativamente de tres tipos de recubrimientos de zinc con diferentes combinaciones de $\mathrm{Zn}, \mathrm{Al}, \mathrm{Mg}$ y $\mathrm{Cu}(\mathrm{Zn}-0,1 \mathrm{Cu}(\mathrm{G}), \mathrm{Zn}-5 \mathrm{Al}-0,1 \mathrm{Cu}$ (ZA) y Zn-5Al-1Mg-0,1Cu (ZAM)). Realizaron pruebas de cámara de niebla salina y electroquímicos en una solución acuosa de 5 y 3,5\% de $\mathrm{NaCl}$, respectivamente, para acceder al comportamiento de corrosión de los recubrimientos.

En la Figura 10 (a) y (b) se observan las curvas de polarización de los recubrimientos, preparados superficialmente y después de cinco días de ensayos en la cámara de niebla salina, respectivamente. Se observaron algunos cambios en los parámetros del proceso de corrosión. El recubrimiento ZAM en la curva de polarización mostró una región, en el rango de $-1,2 \mathrm{~V}$ a $-1,5 \mathrm{~V}$ (Figura 9b), que mantiene la densidad de corriente relativamente constante independientemente de la variación de potencial, lo que indica que la reacción catódica del recubrimiento ZAM se inhibió en este rango de potencial, debido a la precipitación de productos de corrosión en el área catódica, que actúan como inhibidores, aumentando la impedancia de la superficie del recubrimiento. 
Hernández y Suárez / Efecto de la composición química del baño en la microestructura y resistencia a la corrosión de los recubrimientos de zinc por inmersión en caliente: Una revisión

Tabla 2. Técnicas y ambientes para evaluar el comportamiento a la corrosión de los recubrimientos zinc

\begin{tabular}{|c|c|c|c|}
\hline $\begin{array}{l}\text { Tipo de } \\
\text { recubrimiento }\end{array}$ & $\begin{array}{l}\text { Ambiente } \\
\text { corrosivo }\end{array}$ & $\begin{array}{l}\text { Técnica de } \\
\text { evaluación }\end{array}$ & Ref. \\
\hline $\begin{array}{c}\mathrm{Zn} \\
\mathrm{Zn}-4,9 \mathrm{Al}\end{array}$ & $\begin{array}{l}5 \% \mathrm{NaCl} \text { en } \\
\text { laboratorio } \\
3 \% \mathrm{NaCl} \text { en } \\
\text { laboratorio }\end{array}$ & $\begin{array}{c}\text { Cámara niebla } \\
\text { salina } \\
\text { Polarización } \\
\text { Potenciodinámica }\end{array}$ & {$[26]$} \\
\hline $\begin{array}{l}\mathrm{Zn}-5 \mathrm{Al} \\
\mathrm{Zn}-55 \mathrm{Al}\end{array}$ & $\begin{array}{c}\text { Agua de mar real } \\
(78 \% \mathrm{NaCl}) \\
\text { Agua de mar sintética } \\
\qquad(68 \% \mathrm{NaCl})\end{array}$ & $\begin{array}{l}\text { Mediciones de } \\
\text { potenciales }\end{array}$ & {$[27]$} \\
\hline $\begin{array}{c}\mathrm{Zn} \\
\mathrm{Zn}-5 \mathrm{Al}\end{array}$ & $\begin{array}{l}3 \% \mathrm{NaCl} \text { en } \\
\text { laboratorio }\end{array}$ & $\begin{array}{c}\text { Cámara niebla } \\
\text { salina } \\
\text { Polarización } \\
\text { Potenciodinámica }\end{array}$ & [28] \\
\hline $\begin{array}{c}\mathrm{Zn}-0,2 \mathrm{Al} \\
\mathrm{Zn}-2 \mathrm{Al}-2 \mathrm{Mg}\end{array}$ & $\begin{array}{l}5 \% \mathrm{NaCl} \text { en } \\
\text { laboratorio }\end{array}$ & $\begin{array}{l}\text { Cámara niebla } \\
\text { salina }\end{array}$ & {$[11]$} \\
\hline $\begin{array}{c}\mathrm{Zn} \\
\mathrm{Zn}-5 \mathrm{Al} \\
\mathrm{Zn}-55 \mathrm{Al}\end{array}$ & $\begin{array}{l}\text { Atmosférica } \\
\text { en campo }\end{array}$ & $\begin{array}{l}\text { Ensayos de } \\
\text { campo }\end{array}$ & [29] \\
\hline $\begin{array}{c}\mathrm{Zn} \\
\mathrm{Zn}-55 \mathrm{Al}\end{array}$ & $\begin{array}{c}\text { Atmosférica } \\
\text { de campo } \\
\text { Cámara } \\
\text { neblina salina } \\
\text { y de } \mathrm{SO}_{2} \text { en } \\
\text { laboratorio }\end{array}$ & $\begin{array}{l}\text { Espectroscopía } \\
\text { de impedancia } \\
\text { electroquímica }\end{array}$ & [30] \\
\hline $\mathrm{Zn}-55 \mathrm{Al}$ & $\begin{array}{c}0,5 \mathrm{M} \mathrm{NaCl} \\
0,5 \mathrm{M} \mathrm{Na}_{2} \mathrm{SO}_{4} \\
0,5 \mathrm{M} \mathrm{NaClO}_{4}\end{array}$ & $\begin{array}{l}\text { Espectroscopía } \\
\text { de impedancia } \\
\text { electroquímica }\end{array}$ & {$[31]$} \\
\hline $\begin{array}{c}\mathrm{Zn} \\
\mathrm{Zn}-5 \mathrm{Al}\end{array}$ & $\begin{array}{l}5 \% \text { solución } \\
\text { amoniacal }\end{array}$ & $\begin{array}{l}\text { Ensayo corrosión } \\
\text { cíclica }\end{array}$ & {$[32]$} \\
\hline $\begin{array}{c}\mathrm{Zn} / \mathrm{Zn}-6 \mathrm{Al} \\
\mathrm{Zn} / \mathrm{Zn}-6 \mathrm{Al}-0,5 \mathrm{Mg}-0,1 \mathrm{Si}\end{array}$ & $\begin{array}{l}5 \% \mathrm{NaCl} \text { en } \\
\text { laboratorio }\end{array}$ & Inmersión & {$[12]$} \\
\hline $\begin{array}{c}\mathrm{Zn} \\
\mathrm{Zn} / \mathrm{Zn}-7 \mathrm{Al}\end{array}$ & $\begin{array}{l}\text { Agua de mar } \\
\text { real }\end{array}$ & Inmersión & [13] \\
\hline
\end{tabular}

La Figura 11 (a) y (b) muestra los espectros EIS para los recubrimientos G, ZA y ZAM. Las formas de los gráficos de Nyquist (a) y los diagramas de ángulo de fase de Bode (b) de los tres recubrimientos son similares. Las gráficas de Nyquist consisten en un semicírculo a alta frecuencia y un círculo a baja frecuencia. Los diagramas de ángulo de fase de Bode exhiben dos crestas de onda, lo que indica que los tres recubrimientos pueden tener mecanismos de corrosión similares. Mientras que el recubrimiento ZA tiene una mayor impedancia principalmente en el rango de alta frecuencia $(10 \mathrm{~Hz} \sim 100000 \mathrm{~Hz})$ que los otros dos recubrimientos, el recubrimiento $\mathrm{G}$ tiene una impedancia relativamente más alta a baja frecuencia $(0.01 \mathrm{~Hz} \sim 10 \mathrm{~Hz})$ que los recubrimientos ZA y ZAM. 


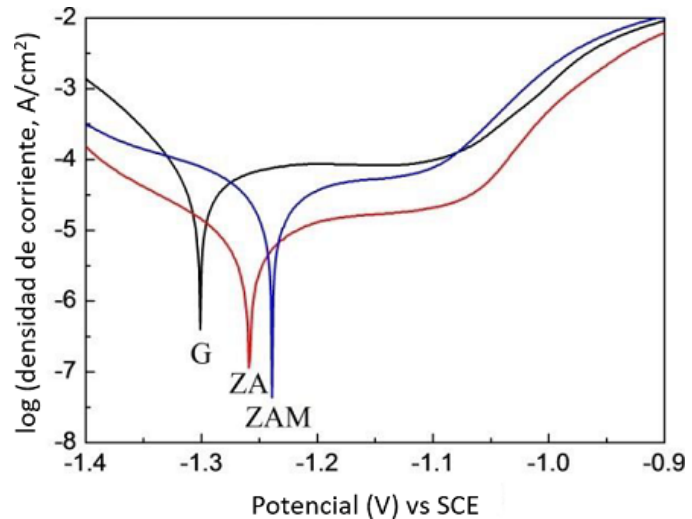

(a)

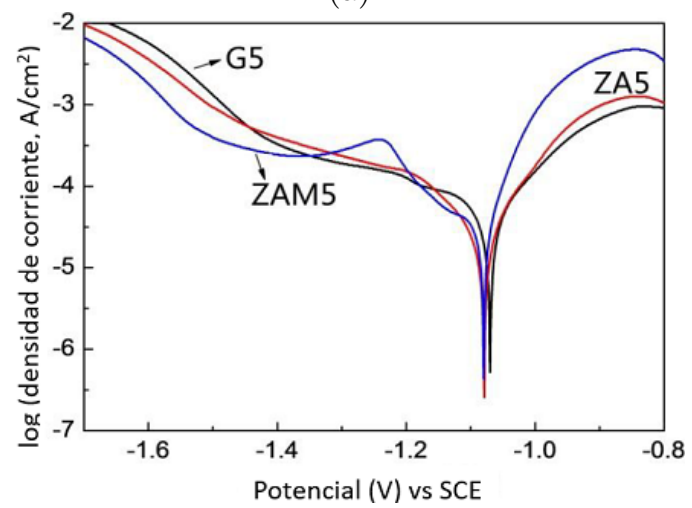

(b)

Figura 10. Curvas de polarización potenciodinámica para los recubrimientos de cinc; (a) preparados superficialmente; (b) después de 5 días del ensayo de cámara de niebla salina $[10]$.

Un valor de impedancia más alto normalmente indica una velocidad de corrosión más baja del material. La impedancia del recubrimiento ZAM es más baja que otras en esta etapa, lo que puede deberse a la adición de $\mathrm{Mg}$ menos noble, lo cual hará que reaccione primero cuando el recubrimiento ZAM esté en una solución de $\mathrm{NaCl}$, contribuyendo a la alta densidad de corriente de corrosión en la etapa inicial.

La naturaleza protectora del recubrimiento ZAM puede atribuirse a la corrosión inicial de las fases ricas en $\mathrm{Mg}$. Los productos de corrosión de $\mathrm{Zn}, \mathrm{Al}$ y $\mathrm{Mg}$ se aglomeran en el área catódica, que actúan como inhibidores, bloqueando las rutas de corrosión (las microrrutas para la difusión de $\mathrm{O}_{2}$ y $\mathrm{H}_{2} \mathrm{O}$ ) a lo largo de los límites de grano de los cristales de $\mathrm{Zn}$, y aumentando la impedancia del recubrimiento, por lo tanto, el proceso de corrosión general del recubrimiento ZAM se retrasa.

Tal como se indicó anteriormente, el comportamiento a la corrosión depende de muchos factores y uno de los más estudiados son los referidos a los parámetros de operación del proceso por inmersión en caliente. Por ejemplo, Elvins et al. [14] estudiaron el efecto de la velocidad de enfriamiento sobre la microestructura y la resistencia a la corrosión del recubri- miento Galfan ${ }^{\circledR}(\mathrm{Zn}-4,5 \mathrm{Al})$ sobre el acero. Aplicaron tres velocidades de enfriamiento diferentes.

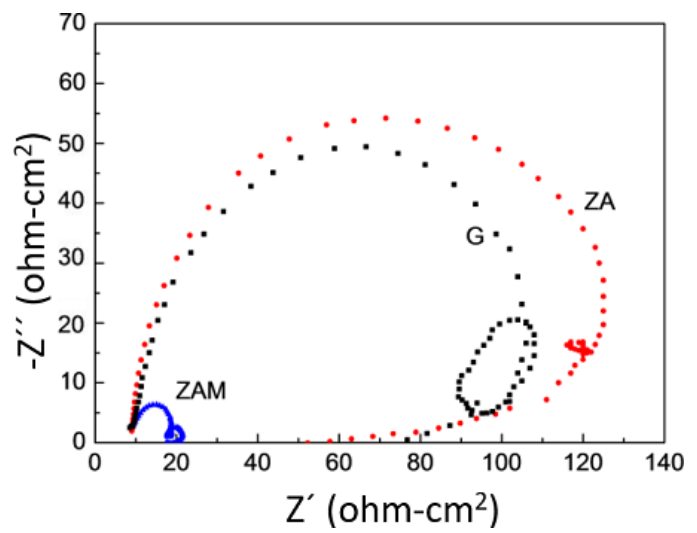

(a)

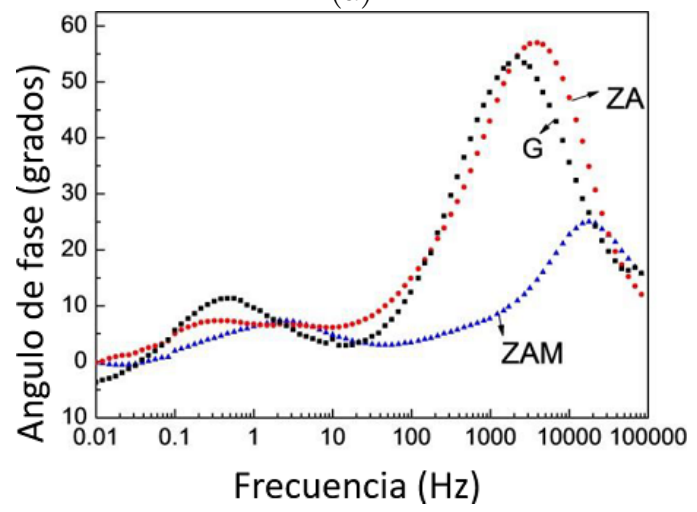

(b)

Figura 11. Espectroscopía de impedancia electroquímica de los recubrimientos de cinc. Diagrama de Nyquist (a). Diagrama de Bode (b). [10]

El aumento en la velocidad de enfriamiento no alteró significativamente el volumen de la fracción del zinc primario; sin embargo, el tamaño y el número de las dendritas primarias de zinc fueron alteradas. Las muestras enfriadas rápidamente contenían pequeñas, pero numerosas, dendritas y en las de enfriamiento lento había menos dendritas de mayor tamaño. La cantidad de dendritas de zinc influye en el proceso de corrosión ya que es la fase que se corroe preferentemente, por lo cual resulta en una mayor área anódica y consecuentemente una mayor velocidad de corrosión a medida que aumenta la velocidad de enfriamiento. En la Figura 12 se observa la corrosión preferencial de las dendritas de zinc respecto al eutéctico.

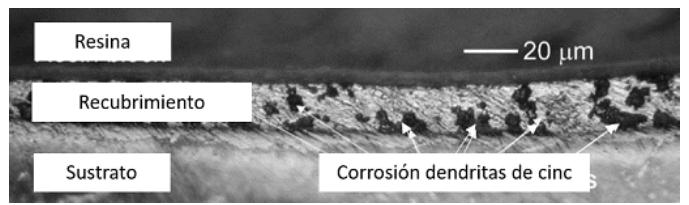

Figura 12. Microestructura del recubrimiento Zn-4,5Al donde se observa el ataque preferencial a las dendritas de zinc. [25] 
Otra forma de estudiar mecanismos y entender de una mejor manera el comportamiento a la corrosión de los recubrimientos de zinc es el utilizar técnicas de análisis a los productos de corrosión para proponer mecanismos del proceso, tal como el trabajo realizado por Carbucicchio et al. [33], donde estudiaron los productos de corrosión formado en tuberías de acero galvanizado expuesto por dos años a agua caliente, mediante técnicas metalográficas, de difracción de rayos $\mathrm{X}$, así como las técnicas espectroscópicas Mössbauer y Raman. Los resultados obtenidos permitieron establecer que el proceso corrosivo fue causado por un exceso de la temperatura de servicio, ocurriendo una inversión de la polaridad electroquímica entre el zinc y el hierro, creciendo tubérculos de productos de corrosión en las zonas descincificadas de la pared de la tubería hasta perforarse. Los compuestos presentes en los productos de corrosión permitieron sugerir un mecanismo del proceso.

Por otra parte, también es importante mencionar que los procesos corrosivos se ven afectados por algunos esfuerzos mecánicos, o algún elemento específico como el hidrógeno que contribuye a acrecentar el proceso corrosivo, por lo cual también hay investigaciones que evalúan comportamientos sinérgicos de la corrosión con otros tipos de desgaste o esfuerzos mecánicos. En este sentido, Reumont et al. [34], presentaron un estudio para investigar el papel de la microestructura en la susceptibilidad a la corrosión asistida por esfuerzos (SCC) en una solución acuosa de cloruro de sodio de un acero galvanizado. Se efectuaron ensayos de deformación en una solución acuosa de $\mathrm{NaCl}$ al $3 \%$. El comportamiento general del acero galvanizado es dúctil. Existe una transición de dúctil a frágil para una velocidad de deformación muy particular en este ambiente. Poca resistencia mecánica del recubrimiento y la reacción de hidrólisis del zinc es necesaria para conducir a la fragilidad, la cual puede estar asociada con un proceso de fragilidad por hidrógeno. La formación de compuestos intermetálicos puede producir un recubrimiento de microestructura resistente al SCC.

Un trabajo similar efectuó Sacco et al. [31], donde evaluaron el comportamiento de chapas de acero con recubrimientos de zinc convencional y con $\mathrm{Zn}-55 \mathrm{Al}$ traccionadas uniaxialmente y expuestas a soluciones $0,5 \mathrm{M}$ $\mathrm{NaCl} ; 0,5 \mathrm{M} \mathrm{Na}_{2} \mathrm{SO}_{4}$; y $0,5 \mathrm{M} \mathrm{NaClO}_{4}$. Las muestras fueron deformadas hasta alcanzar alargamientos de 1,$3 ; 3,75 ; 6,25 ; 7,5$ y $12,5 \%$. Estas y otras muestras $\sin$ deformar fueron sometidas a ensayos electroquímicos y medidas de impedancia electroquímica. Los resultados obtenidos permitieron inferir que existen notorias diferencias en la cinética de las reacciones de corrosión que tienen lugar en la superficie de ambos tipos de recubrimiento y que para ambos sistemas acero/recubrimiento metálico dicha cinética es función no solo del grado de deformación aplicado sino también de la composición del medio agresivo considerado.
Rico y Carrasquero [4] efectuaron una revisión donde establecen que la composición química de los baños galvanizados influye fuertemente en las características microestructurales de los recubrimientos y en su comportamiento mecánico. Esta aseveración, junto con los estudios reportados en este trabajo establece que el comportamiento a la corrosión también se ve afectado por la adición de elementos al baño de zinc; además, esto se sustenta en otros trabajos donde se estudiaron los efectos sinérgicos de la corrosión con el comportamiento mecánico [31,34-38].

Tal como se mencionó anteriormente en la primera sección de este trabajo, otras investigaciones que se destacan con el fin de mejorar las propiedades a la corrosión de los recubrimientos de zinc, es realizar procesos de doble inmersión, que consisten en sumergir el acero consecutivamente, en dos baños líquidos con diferentes composiciones químicas; la primera inmersión se realiza en baño de galvanizado convencional y la segunda en baño de una aleación base zinc con otros elementos, como el aluminio, el magnesio y el silicio. En este sentido, Tanaka et al. [12] demostraron que la doble inmersión tiene ventajas tales como la buena adhesión del recubrimiento y la viabilidad de controlar mejor el espesor y la microestructura del recubrimiento, por lo cual también mejora su resistencia a la corrosión. Para establecer la mejora del comportamiento a la corrosión efectuó ensayos estandarizados en laboratorio.

Tachibana y colaboradores [13], también aplicando un proceso de doble inmersión, determinaron que los recubrimientos efectuados por este método presentan un mejor comportamiento a la corrosión que los fabricados por métodos convencionales. Estos resultados se obtuvieron en pruebas realizadas en un medioambiente salino real.

Es importante destacar, que aun cuando se han realizado algunas investigaciones acerca de la aplicación de los procesos de doble inmersión, no hay información certera acerca de los mecanismos que permiten establecer la mejora en la resistencia a la corrosión de estos procesos en comparación con otros métodos convencionales; sin embargo, se estima que la adición a los baños de elementos generalmente más activos que el zinc, tales como el magnesio o el aluminio, permiten la formación de capas pasivas que retardan el proceso corrosivo.

\section{Conclusiones}

La composición química de los baños galvanizados influye en las características microestructurales de los recubrimientos.

El aumento de la resistencia a la corrosión de los recubrimientos de zinc por inmersión en caliente se orienta a cambiar la composición química de los baños, 
con el fin de ampliar la aplicabilidad de estos recubrimientos en ambientes más agresivos como aquellos que contienen cloruros.

La mejora de la resistencia a la corrosión de los recubrimientos de zinc se produce por la adición a los baños de elementos generalmente más activos que el zinc, tales como el magnesio o el aluminio que permiten la formación de capas pasivas que retardan el proceso corrosivo.

Los estudios de los recubrimientos de $\mathrm{Zn}-\mathrm{Al}-\mathrm{Mg}$ han reportado comportamientos a la corrosión del orden de 10 a 20 veces respecto al recubrimiento de $\mathrm{Zn}$ y de 2 a 5 respecto a los de $\mathrm{Zn}-\mathrm{Al}$.

\section{Referencias}

[1] J. Cervantes, A. Barba, M. Hernández, J. Salas, J. Espinoza, C. Dénova, G. Torres-Villaseñor, A. Conde, A. Covelo, and R. Valdez, "Obtención y caracterización de recubrimientos $\mathrm{Zn}-\mathrm{Al}-\mathrm{Cu}$ por inmersión en caliente sobre aceros de bajo carbono," Revista de Metalurgia, vol. 49, no. 5, pp. 351-359, 2013. [Online]. Available: https://doi.org/10.3989/revmetalm.1316

[2] L. Shiwei, G. Bo, T. Ganfeng, H. Yi, H. Liang, and Y. Shaohua, "Study on the corrosion mechanism of zn-5al-0.5mg-0.08si coating," Journal of Metallurgy, vol. 2011, Article ID 917469, pp. 1-6, 2011. [Online]. Available: https://doi.org/10.1155/2011/917469

[3] A. R. Marder, "The metallurgy of zinc-coated steel," Progress in Materials Science, vol. 45, no. 3, pp. 191-271, 2000. [Online]. Available: https://doi.org/10.1016/S0079-6425(98)00006-1

[4] Y. Rico and E. Carrasquero, "Efecto de la composición química en el comportamiento mecánico de recubrimientos galvanizados por inmersión en caliente: una revisión," Ingenius, no. 18 , pp. 30-39, 2017. [Online]. Available: http://dx.doi.org/10.17163/ings.n18.2017.04

[5] American Galvanizers Association, "Hot-dip galvanizing for corrosion protection a specifier's guide," 2012. [Online]. Available: https://bit.ly/2pGEzeb

[6] Y. Rico and J. Hernández, "Influencia de la velocidad de enfriamiento sobre la microestructura y comportamiento a la corrosión de recubrimientos Zn-7Al por inmersión en caliente sobre acero," Revista Latinoamericana de Metalurgia y Materiales, vol. 35, pp. 269-275, 12 2015. [Online]. Available: https://bit.ly/2OaV0sy

[7] S. D. Cramer and J. Bernard S. Covino, "Corrosion: Fundamentals, testing, and protection,"
ASM International. The Materials Information Society, Tech. Rep., 2003. [Online]. Available: https://bit.ly/2pGH6Fd

[8] Y. Rico and E. J. Carrasquero, "Microstructural evaluation of double-dip galvanized coatings on carbon steel," MRS Advances, vol. 2, no. 62 , pp. 3917-3923, 2017. [Online]. Available: https://doi.org/10.1557/adv.2017.608

[9] P. Volovitch, T. N. Vu, C. Allély, A. A. Aal, and K. Ogle, "Understanding corrosion via corrosion product characterization: Ii. role of alloying elements in improving the corrosion resistance of Zn-Al-Mg coatings on steel," Corrosion Science, vol. 53, no. 8, pp. 2437-2445, 2011. [Online]. Available: https://doi.org/10.1016/j.corsci.2011.03.016

[10] C. Yao, S. L. Tay, J. H. Yang, T. Zhu, and W. Gao, "Hot dipped Zn-Al-Mg-Cu coating with improved mechanical and anticorrosion properties," International Journal of Electrochemical Science, vol. 9, pp. 7083-7096, 2014. [Online]. Available: https://bit.ly/2XChPZq

[11] S. Schuerz, M. Fleischanderl, G. H. Luckeneder, K. Preis, T. Haunschmied, G. Mori, and A. C. Kneissl, "Corrosion behaviour of $\mathrm{Zn}-\mathrm{Al}-\mathrm{Mg}$ coated steel sheet in sodium chloride-containing environment," Corrosion Science, vol. 51, no. 10 , pp. 2355-2363, 2009. [Online]. Available: https://doi.org/10.1016/j.corsci.2009.06.019

[12] J. Tanaka, K. Ono, S. Hayashi, K. Ohsasa, and T. Narita, "Effect of Mg and Si on the microstructure and corrosion behavior of Zn-Al hot dip coatings on low carbon steel," ISIJ International, vol. 42, no. 1, pp. 80-85, 2002. [Online]. Available: https://doi.org/10.2355/isijinternational.42.80

[13] K. Tachibana, Y. Morinaga, and M. Mayuzumi, "Hot dip fine zn and zn-al alloy double coating for corrosion resistance at coastal area," Corrosion Science, vol. 49, no. 1, pp. 149157, 2007, progress in Corrosion Research in Commemoration of Centenary of Birth of Professor Go Okamoto. [Online]. Available: https://doi.org/10.1016/j.corsci.2006.05.015

[14] J. Elvins, J. A. Spittle, and D. A. Worsley, "Microstructural changes in zinc aluminium alloy galvanising as a function of processing parameters and their influence on corrosion," Corrosion Science, vol. 47, no. 11 , pp. $2740-2759,2005$. [Online]. Available: https://doi.org/10.1016/j.corsci.2004.11.011

[15] D. Thierry and N. LeBozec, "Corrosion products formed on confined hot-dip galvanized steel in accelerated cyclic corrosion tests," CORROSION, 
vol. 65, no. 11, pp. 718-725, 2009. [Online]. Available: https://doi.org/10.5006/1.3319098

[16] M. Dutta, A. K. Halder, and S. B. Singh, "Morphology and properties of hot dip Zn-Mg and Zn-Mg-Al alloy coatings on steel sheet," Surface and Coatings Technology, vol. 205, no. 7, pp. 2578-2584, 2010. [Online]. Available: https://doi.org/10.1016/j.surfcoat.2010.10.006

[17] N. LeBozec, D. Thierry, D. Persson, C. K. Riener, and G. Luckeneder, "Influence of microstructure of zinc-aluminium-magnesium alloy coated steel on the corrosion behavior in outdoor marine atmosphere," Surface and Coatings Technology, vol. 374, pp. 897-909, 2019. [Online]. Available: https://doi.org/10.1016/j.surfcoat.2019.06.052

[18] P. Volovitch, C. Allely, and K. Ogle, "Understanding corrosion via corrosion product characterization: I. Case study of the role of $\mathrm{Mg}$ alloying in Zn-Mg coating on steel," Corrosion Science, vol. 51, no. 6, pp. 1251-1262, 2009. [Online]. Available: https://doi.org/10.1016/j.corsci.2009.03.005

[19] S. Schürz, G. H. Luckeneder, M. Fleischanderl, P. Mack, H. Gsaller, A. C. Kneissl, and G. Mori, "Chemistry of corrosion products on $\mathrm{Zn}-\mathrm{Al}-\mathrm{Mg}$ alloy coated steel," Corrosion Science, vol. 52, no. 10, pp. 3271-3279, 2010. [Online]. Available: https://doi.org/10.1016/j.corsci.2010.05.044

[20] P. Sixtos, J. Cervantes, A. Barba, J. Roviroza, E. Garduño, R. Valdez, M. Trujillo, J. González, J. Salas, and G. Torres, "Evaluación de recubrimientos de aleaciones $\mathrm{Zn}-\mathrm{Al}-\mathrm{Cu}$ obtenidos por inmersion en caliente y por via termoquimica," in XV Congreso Internacional Anual de la SOMIM, del 23 al 25 de septiembre del 2009. Cd. Obregón, Sonora, México, 2009, pp. 678-682. [Online]. Available: https://bit.ly/34gcWaQ

[21] A. Barba, J. Cervantes, R. González, D. Hernández, V. Hernández, R. Valdez, A. Covelo, and M. Hernández, "Influencia de la adición de pequeñas cantidades de $\mathrm{Ni}$ a baños de galvanizado por inmersión en caliente, en las propiedades de aceros al carbono galvanizados," in $X X I V$ Congreso Iinternacional Anual de la SOMIM 19 al 21 de septiembre de 2018, Campeche, México, 2018. [Online]. Available: https://bit.ly/37xBQVt

[22] C. M. Cotell, J. A. Sprague, and J. F.A. Smidt, ASM Handbook, Volume 5: Surface Engineering, ASM International, Ed., 1994. [Online]. Available: https://bit.ly/35qcdUQ

[23] Y. Li, "Formation of nano-crystalline corrosion products on $\mathrm{Zn}-\mathrm{Al}$ alloy coating exposed to seawater," Corrosion Science, vol. 43, no. 9, pp. 1793-1800, 2001. [Online]. Available: https://doi.org/10.1016/S0010-938X(00)00169-4

[24] M. S. Azevedo, C. Allély, K. Ogle, and P. Volovitch, "Corrosion mechanisms of $\mathrm{Zn}(\mathrm{Mg}$, $\mathrm{Al})$ coated steel in accelerated tests and natural exposure: 1. The role of electrolyte composition in the nature of corrosion products and relative corrosion rate," Corrosion Science, vol. 90, pp. 472-481, 2015. [Online]. Available: https://doi.org/10.1016/j.corsci.2014.05.014

[25] N. C. Hosking, M. A. Ström, P. H. Shipway, and C. D. Rudd, "Corrosion resistance of zinc-magnesium coated steel," Corrosion Science, vol. 49, no. 9, pp. 3669-3695, 2007. [Online]. Available: https://doi.org/10.1016/j.corsci.2007.03.032

[26] M. Manna, G. Naidu, N. Rani, and N. Bandyopadhyay, "Characterisation of coating on rebar surface using hot-dip Zn and Zn-4.9Al-0.1 misch metal bath," Surface and Coatings Technology, vol. 202, no. 8, pp. 1510-1516, 2008. [Online]. Available: https://doi.org/10.1016/j.surfcoat.2007.07.001

[27] A. P. Yadav, H. Katayama, K. Noda, H. Masuda, A. Nishikata, and T. Tsuru, "Effect of al on the galvanic ability of $\mathrm{Zn}-\mathrm{Al}$ coating under thin layer of electrolyte," Electrochimica Acta, vol. 52, no. 7, pp. 2411-2422, 2007. [Online]. Available: https://doi.org/10.1016/j.electacta.2006.08.050

[28] H. C. Shih, J. W. Hsu, C. N. Sun, and S. C. Chung, "The lifetime assessment of hot-dip $5 \%$ Al-Zn coatings in chloride environments," Surface and Coatings Technology, vol. 150, no. 1, pp. 70-75, 2002. [Online]. Available: https://doi.org/10.1016/S0257-8972(01)01508-0

[29] O. de Rincón, A. Rincón, M. Sánchez, N. Romero, O. Salas, R. Delgado, B. López, J. Uruchurtu, M. Marroco, and Z. Panosian, "Evaluating $\mathrm{Zn}, \mathrm{Al}$ and $\mathrm{Al}-\mathrm{Zn}$ coatings on carbon steel in a special atmosphere," Construction and Building Materials, vol. 23, no. 3, pp. 1465-1471, 2009. [Online]. Available: https: //doi.org/10.1016/j.conbuildmat.2008.07.002

[30] S. Flores and K. Paucar, "Evaluación de sistemas de recubrimientos mediante espectroscopía de impedancia electroquímica," Jornadas SAM CONAMET - Simposio Materia 2003, 2003. [Online]. Available: https://bit.ly/2KIjsPR

[31] E. Sacco, J. Culcasi, C. Eisner, and A. D. Darli, "Efecto de la deformación por tracción sobre la orientación cristalográfica del recubrimiento y la resistencia a la corrosión en CNS de chapas de acero con recubrimientos base 
cinc," Revista de Metalurgia, vol. 38, no. 6, pp. 403-409, 2002. [Online]. Available: https: //doi.org/10.3989/revmetalm.2002.v38.i6.425

[32] H. Fujisawa, R. Kaneko, and H. Ishikawa, "Hot-Dip Zn-5 \% Al alloy-coated Steel Sheets JFE ECOGAL ®," JFE Technical Report, no. 14, pp. 41-45, 2009. [Online]. Available: https://bit.ly/335wY6I

[33] M. Carbucicchio, R. Ciprian, F. Ospitali, and G. Palombarini, "Morphology and phase composition of corrosion products formed at the zinc-iron interface of a galvanized steel," Corrosion Science, vol. 50, no. 9, pp. 2605-2613, 2008. [Online]. Available: https://doi.org/10.1016/j.corsci.2008.06.007

[34] G. Reumont, J. B. Vogt, A. Iost, and J. Foct, "The effects of an Fe-Zn intermetalliccontaining coating on the stress corrosion cracking behavior of a hot-dip galvanized steel," Surface and Coatings Technology, vol. 139, no. 2, pp. 265-271, 2001. [Online]. Available: https://doi.org/10.1016/S0257-8972(01)01017-9

[35] N. Michailidis, F. Stergioudi, G. Maliaris, and A. Tsouknidas, "Influence of galvanization on the corrosion fatigue performance of high-strength steel," Surface and Coatings Technology, vol. 259, pp. 456-464, 2014. [Online]. Available: https://doi.org/10.1016/j.surfcoat.2014.10.049

[36] K. A. Yasakau, S. Kallip, A. Lisenkov, M. G. S. Ferreira, and M. L. Zheludkevich, "Initial stages of localized corrosion at cut-edges of adhesively bonded $\mathrm{Zn}$ and $\mathrm{Zn}$ Al-Mg galvanized steel," Electrochimica Acta, vol. 211, pp. 126-141, 2016. [Online]. Available: https://doi.org/10.1016/j.electacta.2016.06.045

[37] E. Carrasquero, Y. Rico, and J. Minchala, "Evaluación de propiedades mecánicas en recubrimientos galvanizados por doble inmersión en caliente sobre acero al carbono," INGENIUS, no. 22, pp. 80-89, 2019. [Online]. Available: https://doi.org/10.17163/ings.n22.2019.08

[38] K. Zhang, R. Song, and Y. Gao, "Corrosion behavior of hot-dip galvanized advanced high strength steel sheet in a simulated marine atmospheric environment," International Journal of Electrochemical Science, no. 14, pp. 1488-1499, 2019. [Online]. Available: http://doi.org/10.20964/2019.02.13 